\title{
Actionable Mutations in Plasma Cell-Free DNA in Patients with Advanced Cancers Referred for Experimental Targeted Therapies
}

\author{
Filip Janku ${ }^{1}$, Philipp Angenendt ${ }^{2}$, Apostolia M. Tsimberidou ${ }^{1}$, Siqing Fu $^{1}$ \\ Aung Naing ${ }^{1}$, Gerald S. Falchook ${ }^{1}$, David S. Hong ${ }^{1}$, Veronica R. Holley ${ }^{1}$, \\ Goran Cabrilo $^{1}$, Jennifer J. Wheler ${ }^{1}$, Sarina A. Piha-Paul ${ }^{1}$, Ralph G. Zinner ${ }^{1}$, Agop \\ Y. Bedikian ${ }^{3}$, Michael J. Overman ${ }^{4}$, Bryan K. Kee ${ }^{4}$, Kevin B. Kim ${ }^{3}$, E. Scott Kopetz ${ }^{4}$, \\ Rajyalakshmi Luthra5, Frank DiehI², Funda Meric-Bernstam', Razelle Kurzrock ${ }^{1,6}$ \\ ${ }^{1}$ Department of Investigational Cancer Therapeutics (Phase I Clinical Trials Program), The University of Texas MD Anderson \\ Cancer Center, Houston, US \\ ${ }^{2}$ Sysmex Inostics GmbH, Hamburg, Germany \\ ${ }^{3}$ Department of Melanoma Medical Oncology, The University of Texas MD Anderson Cancer Center, Houston, US \\ ${ }^{4}$ Department of Gastrointestinal Medical Oncology, The University of Texas MD Anderson Cancer Center, Houston, US \\ ${ }^{5}$ Molecular Diagnostic Laboratory, The University of Texas MD Anderson Cancer Center, Houston, US \\ ${ }^{6}$ Moores Cancer Center, The University of California San Diego, La Jolla, Califorina
}

Correspondence to:

Filip Janku, e-mail: fjanku@mdanderson.org

Keywords: EGFR, BRAF, KRAS, PIK3CA, cell-free DNA

Received: December 16, $2014 \quad$ Accepted: February 11, $2015 \quad$ Published: February 25, 2015

\section{ABSTRACT}

Cell-free (cf) DNA in the plasma of cancer patients offers an easily obtainable source of biologic material for mutation analysis. Plasma samples from 157 patients with advanced cancers who progressed on systemic therapy were tested for 21 mutations in BRAF, EGFR, KRAS, and PIK3CA using the BEAMing method and results were compared to mutation analysis of archival tumor tissue from a CLIAcertified laboratory obtained as standard of care from diagnostic or therapeutic procedures.Results were concordant for archival tissue and plasma cfDNA in $91 \%$ cases for BRAF mutations (kappa $=0.75,95 \%$ confidence interval [CI] $0.63-$ 0.88 ), in $99 \%$ cases for EGFR mutations (kappa $=0.90,95 \%$ CI $0.71-1.00$ ), in $83 \%$ cases for KRAS mutations (kappa $=0.67,95 \% \mathrm{CI} 0.54-0.80$ ) and in 91\% cases for PIK3CA mutations (kappa = 0.65, 95\% CI $0.46-0.85$ ). Patients $(n=41)$ with $>1 \%$ of KRAS mutant cfDNA had a shorter median survival compared to 20 patients with $\angle /=1 \%$ of KRAS mutant DNA ( 4.8 vs. 7.3 months, $p=0.008$ ). Similarly, 67 patients with $>1 \%$ of mutant cfDNA (BRAF, EGFR, KRAS, or PIK3CA) had a shorter median survival compared to 33 patients with $\angle /=1 \%$ of mutant cfDNA (5.5 vs. 9.8 months, $p=0.001$ ), which was confirmed in multivariable analysis.

\section{INTRODUCTION}

The discovery of oncogenic mutations has expanded our understanding of the mechanisms of tumorigenesis and led to the development of targeted cancer therapies directed at specific druggable targets. [1-5] Examples include BRAF inhibitors in melanoma harboring BRAF mutations, ABL kinase inhibitors in chronic myelogenous leukemia with $B C R-A B L$ fusion, EGFR tyrosine kinase inhibitors in non-small cell lung cancer (NSCLC) with an EGFR mutation, and others. [1-10] Currently, oncogenic mutations are tested using archival formalin-fixed, paraffin-embedded tumor tissue (FFPE) and its lack of availability is often a limiting factor, precluding mutation analysis. In addition, mutation analysis of primary tumor tissue 
or of an isolated metastasis does not, due to tumor heterogeneity, necessarily reflect the genetic make-up of metastatic disease. [11-15] It has been reported that distinct oncogenic mutations occur in different areas of a primary tumor and that there is a discrepancy in approximately $20-30 \%$ of cases between the mutation status in primary tumor versus distant metastases. [11, 12] In addition, translational studies in EGFR-mutated NSCLC suggested that the cancer genotype can change over time. [13] Sequist et al. demonstrated, in a group of 37 patients with EGFR-mutant NSCLC who had pre-treatment and post-progression tumor biopsies, that some mutations occur and disappear over time. [13] For example, patients who initially responded to an $E G F R$ tyrosine kinase inhibitor developed an $E G F R$ T790M mutation or PIK3CA mutation at the time of disease progression. Consequently, their treatment regimens were changed and the EGFR T790M and $P I K 3 C A$ mutations were no longer detectable in the tumor samples collected a couple of months later, and patients responded again to retreatment with an EGFR tyrosine kinase inhibitor. [13] Having a technique available to elucidate molecular changes potentially underlying drug resistance is of special importance as most patients treated with matched targeted therapies, despite improved response rates and longer progressionfree survival, ultimately develop therapeutic resistance and disease progression.

Cell-free (cf) DNA is released to the circulation from cells undergoing apoptosis or necroptosis in primary or metastatic cancer lesions or in the tumor microenvironment and can be identified in the blood samples of patients with cancer. [16] Unlike performing tissue biopsies, obtaining samples of plasma cfDNA is a noninvasive approach, with less risk to patients at a lower cost. Therefore, in patients with advanced cancer, we investigated whether mutation analysis of plasma-derived cfDNA has an acceptable level of concordance with routine clinical mutation analysis for common oncogenic mutations in BRAF, EGFR, KRAS, and PIK3CA. Tissue testing obtained from prior surgeries and biopsies was performed in the Clinical Laboratory Improvement Amendment (CLIA)-certified Molecular Diagnostic Laboratory at The University of Texas MD Anderson Cancer Center (MD Anderson).

\section{RESULTS}

\section{Patients}

A total of 157 patients with diverse advanced cancers with known FFPE tumor tissue mutation status for mutations in at least one of the selected cancer genes, which included BRAF, EGFR, KRAS, PIK3CA, were enrolled (Table 1). Their median age was 58 years (range, 20 to 84 years) and most patients $(n=118,75 \%)$ were white and men $(n=81,52 \%)$. The most common tumor types were colorectal cancer $(n=68,43 \%)$, melanoma $(n=34,22 \%)$, NSCLC $(n=13,8 \%)$, appendiceal cancer $(n=5,3 \%)$, ovarian cancer $(n=5,3 \%)$ and uterine cancer $(n=5,3 \%)$ (Table 2). The median time between FFPE tumor tissue and plasma collection was 16.5 months (range, 0-144. 7 months). Table 3 provides information about experimental therapies that were given.

\section{Mutations and discrepancy analysis}

Of the 157 patients, 137 were tested for $B R A F$ mutation in tumor and cfDNA samples; 38 (28\%) patients had a $B R A F$ V600 mutation in the FFPE tumor samples and $33(24 \%)$ had $B R A F$ V600 mutations in cfDNA from plasma, with overall agreement between testing modalities in $124(91 \%)$ cases (kappa 0.75, SE 0.07, 95\% confidence interval [CI] 0.63-0.88) with sensitivity 76\% (95\% CI 0.60-0.89), specificity 96\% (95\% CI 0.90-0.99), positive predictive value $88 \%$ (95\% CI $0.72-0.97$ ) and negative predictive value $91 \%$ (95\% CI $0.84-0.96$; Table 1). Of 9 patients (melanoma, $n=6$; colorectal, $n=1$; papillary thyroid, $n=1$; appendiceal cancer, $n=1$ ) with $B R A F$ V600E mutation in the tumor tissue but not in cfDNA, one patient (papillary thyroid cancer) had wtBRAF when the tumor tissue (we used the same block as used for initial tissue testing, if possible) was tested with BEAMing. Three patients (all melanoma) had plasma collection immediately after coming off BRAF or MEK targeted therapy and 1 patient (appendiceal cancer) had plasma collection right after being taken off standard chemotherapy without evidence of disease progression. Of interest, the latter patient had another cfDNA analysis for a $B R A F$ mutation when her disease progressed and at that time $B R A F$ V600Emutant cfDNA was detected. In addition, a patient with colorectal cancer and a $B R A F$ V600E mutation in the tumor tissue, but not in cfDNA, was found to have a KRAS G12D mutation in cfDNA, which was not detected in the tumor tissue. Furthermore, we found a $B R A F$ V $600 \mathrm{~K}$ mutation in cfDNA in 4 patients with wt $B R A F$ in their tumor tissue. Of interest, 2 of these 4 patients (colorectal cancer and NSCLC) also had KRAS G12 mutations in FFPE tumor samples and cfDNA. Finally, a patient with melanoma had a $B R A F$ V600E mutation in the tissue, but a $B R A F \mathrm{~V} 600 \mathrm{~K}$ mutation in cfDNA; however, repeated testing of tumor tissue with BEAMing confirmed a $B R A F$ V600K mutation. We also analyzed whether the amount of $B R A F$-mutant cfDNA correlated with discrepancies between cfDNA and tumor tissue and, indeed, patients with concordant results between cfDNA and tissue had a median of $1.99 \%$ of $B R A F$-mutant cfDNA compared to a median of $0.02 \%$ of $B R A F$-mutant cfDNA in patients with discrepant results $(p=0.001)$.

Of the 157 patients, 79 were tested for $E G F R$ in tumor and cfDNA samples; $5(6 \%)$ patients had $E G F R$ mutations in the FFPE tumor samples and $6(8 \%)$ had 
EGFR mutations in cfDNA from plasma, with overall agreement between testing in $78(99 \%)$ cases (kappa $=1.00$, SE $0.10,95 \%$ CI $0.71-1.00)$ with sensitivity $100 \%(95 \%$ CI $0.48-1.00)$, specificity 99\% (95\% CI $0.93-1.00)$, positive predictive value $83 \%(95 \%$ CI $0.36-0.97)$ and negative predictive value $100 \%$ (95\% CI $0.95-1.00$; Table 1). A patient with Erdheim-Chester disease had an EGFR T790M mutation not previously identified in FFPE tumor samples. Furthermore, 2 patients with EGFR-mutant
NSCLC previously treated with EGFR tyrosine kinase inhibitors also had a second EGFR T790M mutation not previously identified in the FFPE tumor samples, which can plausibly explain why secondary resistance to EGFR targeted therapies occurred. Of interest, a patient treated with erlotinib, who had a biopsy at the time of disease progression revealing an EGFR exon 19 deletion and EGFR T790M mutation, demonstrated no EGFR T790M mutation in cfDNA after 10 months of being taken off of

\section{Table 1: Mutations tested in cfDNA and concordance between tumor tissue and cfDNA}

\begin{tabular}{|c|c|c|}
\hline \multicolumn{3}{|c|}{ Mutations tested } \\
\hline Gene & Exon & Mutation type \\
\hline \multirow[t]{2}{*}{$B R A F$} & 15 & V600E \\
\hline & & V600K \\
\hline \multirow[t]{8}{*}{ EGFR } & 19 & $\Delta$ E746_A750 (2235_2249del15) \\
\hline & & $\Delta$ E746_A750 (2235_2250del15) \\
\hline & & $\Delta$ E746_S752 ins V \\
\hline & & $\Delta$ L747_A750 ins P \\
\hline & & $\Delta$ L747_T751 \\
\hline & & $\Delta$ L747_P753 ins S \\
\hline & 20 & T790M \\
\hline & 21 & L858R \\
\hline \multirow[t]{7}{*}{$K R A S$} & 2 & G12S \\
\hline & & G12R \\
\hline & & G12C \\
\hline & & G12D \\
\hline & & G12A \\
\hline & & G12V \\
\hline & & G13D \\
\hline \multirow[t]{4}{*}{ PIK3CA } & 9 & E542K \\
\hline & & E545K \\
\hline & 20 & H1047R \\
\hline & & H1047L \\
\hline \multicolumn{3}{|c|}{ Concordance between mutation testing of tumor tissue and cfDNA } \\
\hline TESTED $(N=137)$ & $B R A F$ mutation in tumor & $B R A F$ wild-type in tumor \\
\hline$B R A F$ mutation in cfDNA & 29 & 4 \\
\hline$B R A F$ wild-type in cfDNA & 9 & 95 \\
\hline Observed agreements & \multicolumn{2}{|c|}{124 (91\%); Kappa 0.75, SE 0.06; 95 CI\% 0.63-0.88 } \\
\hline Sensitivity & \multicolumn{2}{|l|}{$76 \%(95 \%$ CI $0.60-0.89)$} \\
\hline Specificity & \multicolumn{2}{|l|}{$96 \%(95 \%$ CI $0.90-0.99)$} \\
\hline Positive predictive value & \multicolumn{2}{|l|}{$88 \%(95 \%$ CI $0.72-0.97)$} \\
\hline Negative predictive value & \multicolumn{2}{|l|}{$91 \%(95 \%$ CI $0.84-0.96)$} \\
\hline
\end{tabular}

(Continued) 


\begin{tabular}{|c|c|c|}
\hline TESTED $(N=79)$ & EGFR mutation in tumor & $E G F R$ wild-type in tumor \\
\hline$E G F R$ mutation in cfDNA & 5 & 1 \\
\hline$E G F R$ wild-type in cfDNA & 0 & 73 \\
\hline Observed agreements & \multicolumn{2}{|c|}{78 (99\%); Kappa 0.90, SE 0.10; 95 CI\% 0.71-1.00 } \\
\hline Sensitivity & \multicolumn{2}{|l|}{$100 \%(95 \%$ CI $0.48-1.00)$} \\
\hline Specificity & \multicolumn{2}{|l|}{$99 \%(95 \%$ CI $0.93-1.00)$} \\
\hline Positive predictive value & \multicolumn{2}{|l|}{$83 \%(95 \%$ CI $0.36-0.97)$} \\
\hline Negative predictive value & \multicolumn{2}{|l|}{$100 \%(95 \%$ CI $0.95-1.00)$} \\
\hline $\operatorname{TESTED}(N=121)$ & $K R A S$ mutation in tumor & $K R A S$ wild-type in tumor \\
\hline$K R A S$ mutation in cfDNA & 49 & 8 \\
\hline$K R A S$ wild-type in cfDNA & 12 & 52 \\
\hline Observed agreements & \multicolumn{2}{|c|}{101 (83\%); Kappa 0.67, SE 0.07; 95 CI\% 0.54-0.80 } \\
\hline Sensitivity & \multicolumn{2}{|l|}{$80 \%(95 \%$ CI $0.68-0.89)$} \\
\hline Specificity & \multicolumn{2}{|l|}{$87 \%(95 \%$ CI $0.75-0.94)$} \\
\hline Positive predictive value & \multicolumn{2}{|l|}{$86 \%(95 \%$ CI $0.74-0.94)$} \\
\hline Negative predictive value & \multicolumn{2}{|l|}{$81 \%(95 \%$ CI $0.70-0.90)$} \\
\hline $\operatorname{TESTED}(N=107)$ & $P I K 3 C A$ mutation in tumor & $P I K 3 C A$ wild-type in tumor \\
\hline$P I K 3 C A$ mutation in cfDNA & 12 & 8 \\
\hline$P I K 3 C A$ wild-type in cfDNA & 2 & 85 \\
\hline Observed agreements & \multicolumn{2}{|c|}{97 (91\%); Kappa 0.65, SE 0.10; 95 CI\% 0.46-0.85 } \\
\hline Sensitivity & \multicolumn{2}{|l|}{$86 \%(95 \%$ CI $0.57-0.98)$} \\
\hline Specificity & \multicolumn{2}{|l|}{$91 \%(95 \%$ CI $0.84-0.96)$} \\
\hline Positive predictive value & \multicolumn{2}{|l|}{$60 \%(95 \%$ CI $0.36-0.81)$} \\
\hline Negative predictive value & \multicolumn{2}{|l|}{$98 \%(95 \%$ CI $0.92-1.00)$} \\
\hline
\end{tabular}

Table 2: Clinical characteristics of 157 patients with advanced cancers

Parameter
\begin{tabular}{|l|c|}
\hline Age & Value \\
\hline Median age (range) & $58(20-84)$ \\
\hline Gender & $81(52)$ \\
\hline Men (\%) & $76(48)$ \\
\hline Women (\%) & \\
\hline Ethnicity & $118(75)$ \\
\hline White (\%) & $20(13)$ \\
\hline African-American (\%) & $15(10)$ \\
\hline Hispanic (\%) & $4(3)$ \\
\hline Asian (\%) & \\
\hline Tumor type & $68(43)$ \\
\hline Colorectal cancer (\%) & $34(22)$ \\
\hline Melanoma (\%) & \\
\hline
\end{tabular}

(Continued) 


Parameter
\begin{tabular}{|l|l|}
\hline Non-small cell lung cancer (\%) & Value \\
\hline Ovarian cancer (\%) & $5(3)$ \\
\hline Appendiceal cancer (\%) & $5(3)$ \\
\hline Uterine cancer (\%) & $5(3)$ \\
\hline Breast cancer (\%) & $4(3)$ \\
\hline Non-squamous head and neck cancer (\%) & $4(3)$ \\
\hline Gastroesophageal cancer (\%) & $3(2)$ \\
\hline Papillary thyroid cancer (\%) & $3(2)$ \\
\hline Prostate cancer (\%) & $2(2)$ \\
\hline Soft tissue sarcoma (\%) & $2(2)$ \\
\hline Ampullary cancer (\%) & $1(<1)$ \\
\hline Cholangiocarcinoma (\%) & $1(<1)$ \\
\hline Merkel cell cancer (\%) & $1(<1)$ \\
\hline Small cell lung cancer (\%) & $1(<1)$ \\
\hline Carcinoma of unknown primary (\%) & $1(<1)$ \\
\hline Duodenal cancer (\%) & $1(<1)$ \\
\hline Hepatocellular carcinoma (\%) & $1(<1)$ \\
\hline Squamous head and neck cancer (\%) & $1(<1)$ \\
\hline Erdheim-Chester disease (\%) & $1(<1)$ \\
\hline
\end{tabular}

Table 3: Experimental therapies in patients with $B R A F, E G F R, K R A S$ and $P I K 3 C A$ mutations

\begin{tabular}{|l|c|c|c|c|}
\hline Mutation & Total & Matched therapy & Non-matched therapy & No therapy \\
\hline $\boldsymbol{B R A F}$ (tumor) & 38 & $33^{1}$ & 2 & 3 \\
\hline $\boldsymbol{B R A F}$ (cfDNA) & 33 & $29^{1}$ & 1 & 3 \\
\hline $\boldsymbol{E G F R}$ (tumor) & 5 & $4^{2}$ & 1 & 0 \\
\hline EGFR (cfDNA) & 6 & $4^{2}$ & 2 & 0 \\
\hline KRAS (tumor) & 61 & $0^{3}$ & 47 & 14 \\
\hline KRAS (cfDNA) & 57 & $0^{3}$ & 43 & 14 \\
\hline PIK3CA (tumor) & 14 & $9^{4}$ & 4 & 3 \\
\hline PIK3CA (cfDNA) & 20 & $8^{4}$ & 9 & 1 \\
\hline
\end{tabular}

${ }^{1} \mathrm{BRAF}$ and MEK inhibitors were considered as matched therapies

${ }^{2}$ EGFR inhibitors were considered as matched therapies

${ }^{3}$ There were no matched therapies

${ }^{4} \mathrm{PI} 3 \mathrm{~K} / \mathrm{AKT} / \mathrm{mTOR}$ inhibitors were considered as matched therapies

erlotinib therapy. Because of the low number of patients with EGFR mutations, we did not perform analysis to test associations between the amount of mutant cfDNA and the rate of discrepancies (tumor tissue vs. cfDNA).

Of the 157 patients, 121 were tested for KRAS in tumor and cfDNA samples; 61 (50\%) patients had KRAS G12 or 13 mutations in the FFPE tumor samples and
57 (47\%) had KRAS mutations in cfDNA from plasma with overall agreement between testing in $101(83 \%)$ cases (kappa $=0.67$, SE 0.07, 95\% CI 0.54-0.80) with sensitivity $80 \%$ (95\% CI $0.68-0.89$ ), specificity $87 \%$ ( $95 \%$ CI $0.75-0.94)$, positive predictive value $86 \%$ (95\% CI $0.74-0.94)$ and negative predictive value $81 \%(95 \%$ CI 0.70-0.90; Table 1). Of 12 patients (colorectal, $n=6$; 
appendiceal cancer, $n=2$; NSCLC, $n=2$; duodenal, $n=1$; breast cancer, $n=1$ ) who had KRAS G12 or G13 mutations in the tumor tissue did not have these mutations in their cfDNA. In addition, of 8 patients (colorectal cancer, $n=3$; NSCLC, $n=1$; endometrial cancer, $n=1$; breast cancer, $n=1$; ovarian cancer, $n=1$; melanoma, $n=1$ ) with KRAS G12 or G13 mutation in cfDNA, but not in FFPE tumor samples, 2 patients had KRAS Q61 mutations in FFPE. We also analyzed whether the amount of KRAS-mutant cfDNA correlated with discrepancies between cfDNA and tissue. Patients with concordant results between cfDNA and tissue had a median of $7.46 \%$ $K R A S$-mutant cfDNA compared to a median of $0.55 \%$ $K R A S$-mutant cfDNA in patients with discrepant results $(p=0.048)$.

Of the 157 patients, 107 were tested for $P I K 3 C A$ in tumor and cfDNA samples; in $14(13 \%)$ patients $P I K 3 C A$ mutations were detected in FFPE tumor samples and 20 (19\%) had PIK3CA mutations in cfDNA from plasma with overall agreement between testing in 97 (91\%) cases $(\mathrm{kappa}=0.65$, SE $0.10,95 \%$ CI $0.46-0.85)$ with sensitivity 86\% (95\% CI 0.57-0.98), specificity 91\% (95\% CI $0.84-0.96)$, positive predictive value $60 \%(95 \%$ CI $0.36-0.81)$ and negative predictive value $98 \%(95 \%$ CI 0.92-1.00; Table 1). Two patients (breast cancer, $n=1$; NSCLC, $n=1$ ) had a PIK3CA H1047R mutation their FFPE tumor samples, but not in cfDNA. In contrast, 8 patients (colorectal cancer, $n=4$; squamous cell head and neck, $n=1$; non-squamous head and neck cancer, $n=1$; breast, $n=1$; NSCLC, $n=1$ ) had PIK3CA E542K or E545K mutations in cfDNA but not in FFPE tumor samples. Of interest, 3 of these patients (head and neck, $n=2$; NSCLC, $n=1$ ) were also known to have $E G F R$ mutations and progressed on an EGFR monoclonal antibody or tyrosine kinase inhibitor, suggesting that a PIK3CA mutation could have been a driver of therapeutic resistance. In addition, 2 patients (both with colorectal cancer) had a different PIK3CA mutation in the FFPE tumor samples (Q546P and E545D/M1043L, which were not included in the BEAMing panel). Finally, 4 patients with $P I K 3 C A$ mutations in cfDNA, but not FFPE tumor samples, had simultaneous $K R A S$ mutations in cfDNA and FFPE tumor samples. We also analyzed whether the amount of PIK3CA-mutant cfDNA correlated with discrepancies between cfDNA and tumor tissue and patients with concordant results in cfDNA and tumor tissue had a median of $1.83 \%$ of $P I K 3 C A$-mutant cfDNA compared to a median of $2.61 \%$ of $P I K 3 C A$-mutant cfDNA in patients with discrepant results $(p=0.50)$.

\section{Emergence of low frequency mutations in cfDNA}

In several patients, testing of cfDNA revealed mutations not previously detected in the tumor tissue, which in some of them could have plausibly explained resistance to pertinent targeted therapies. For instance, a patient with NSCLC with wt $B R A F$ and a $K R A S$ G12C mutation in the tissue and cfDNA $(3.80 \%)$ was also found to have a low frequency $B R A F \mathrm{~V} 600 \mathrm{~K}$ mutation in cfDNA $(0.03)$ at the time of disease progression while taking a MEK inhibitor for 3.7 months. A patient with colorectal cancer with wt $B R A F$ and wt $K R A S$ in the primary tumor, who received a cetuximab-based combination for nearly one year had an emergence of a low frequency $B R A F$ V600K mutation in cfDNA $(0.02 \%)$.

Furthermore, a patient with NSCLC and an EGFR L858R mutation found in an original tumor biopsy had cfDNA collection after developing secondary resistance to the EGFR tyrosine kinase inhibitor erlotinib, which in addition to a known EGFR L858R mutation (0.11\%), revealed an EGFR T790M mutation (0.04\%), plausibly explaining secondary resistance to erlotinib. A patient with NSCLC and an EGFR exon 19 deletion from the original biopsy had cfDNA collected after becoming refractory to erlotinib; cfDNA revealed, in addition to an EGFR exon 19 deletion (6.42\%), EGFR T790M (0.65\%) and PIK3CA E545K (0.67\%) mutations not previously identified in the earlier tissue testing, which can credibly explain the emergence of resistance. A patient with NSCLC and an EGFR exon 19 deletion was also found to have a simultaneous EGFR T790M mutation on a tumor biopsy obtained after progression while taking the EGFR tyrosine kinase inhibitor erlotinib; however, unlike with the EGFR exon 19 deletion (12.86\%), EGFR T790M was no longer present in cfDNA obtained 10 months after having been taken off erlotinib.

Furthermore, a patient with a BRAF V600Emutant, wt KRAS colorectal cancer, with a history of early progression to cetuximab-based therapy, was found to have a KRAS G12D mutation (24.39\%) in cfDNA instead, which was not previously detected in the tumor tissue. A patient with wt $K R A S$ in the initial tumor tissue biopsy who had a transient response (4 months) to cetuximab-based therapy was then found to have a KRAS G13D mutation (0.88\%) in cfDNA. Finally, a patient with ovarian cancer and a $P I K 3 C A$ H1047R mutation in an original FFPE tumor sample, who had dramatic but short-lived response to an investigational agent targeting PI3K alpha, was found, in addition to having a PIK3CA H1047R mutation $(0.08 \%)$, a low frequency $K R A S$ G12C mutation $(0.03 \%)$ in cfDNA from plasma collected before initiation of a PI3K inhibitor, which can reasonably explain early therapeutic failure. [21, 22]

Further, a patient with mucoepidermoid carcinoma of the nasal-lacrimal sac with wt PIK3CA and an EGFR exon 18 mutation $(\mathrm{A} 722 \mathrm{~V})$ on an initial biopsy was also found to have a PIK3CA E545K mutation in cfDNA, and the patient was ultimately refractory to treatment with the EGFR tyrosine kinase inhibitor erlotinib. Also, a patient with squamous cell carcinoma of head and neck with wt PIK3CA and an EGFR exon 21 mutation $(\mathrm{H} 835 \mathrm{~L})$ in an initial resected tumor was found to have a PIK3CA E545K mutation $(0.05 \%)$ in cfDNA collected after progressive disease following 3 months of cetuximab, carboplatin and paclitaxel treatment. 


\section{Mutations in cfDNA and overall survival}

Next we investigated whether the amount of mutant cfDNA (percentage compared to wt) had any impact on overall survival (OS). Our strategy was to compare patients with $</=1 \%$ of mutant cfDNA vs. $>1 \%$ cfDNA to make comparable categories. These thresholds were selected based on a $5 \%$ trimmed mean value of mutated cfDNA for all tested genes, which was deemed to be more representative since it was not affected by the number of patients without cfDNA mutations. In addition, these thresholds reflect approximate medians of the percent of mutant DNA for BRAF, EGFR, KRAS and PIK3CA $(1 \%$, $2.7 \%, 3.8 \%$ and $0.5 \%$, respectively).

In 33 patients with $B R A F$ mutations in cfDNA, 16 patients with $<=1 \%$ of $B R A F$-mutant cfDNA had survival rates similar to 17 patients with $>1 \%$ of $B R A F$ mutant cfDNA (8.9 months, $95 \%$ CI $7.3-10.5$ vs. 7.3

A

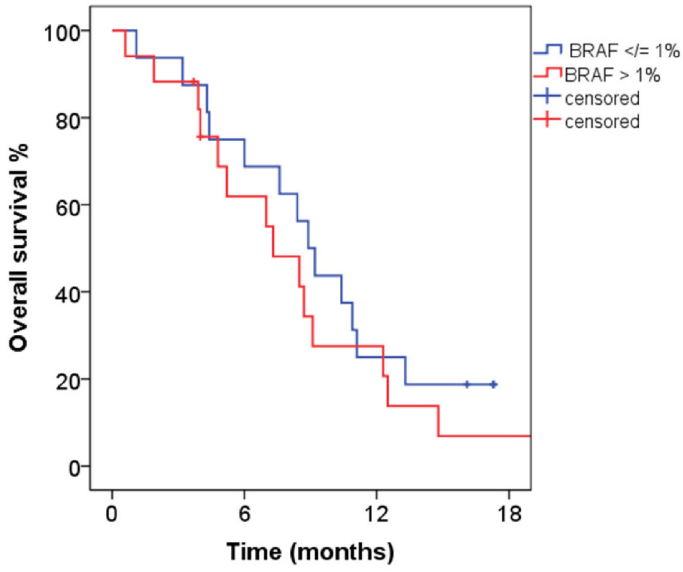

C

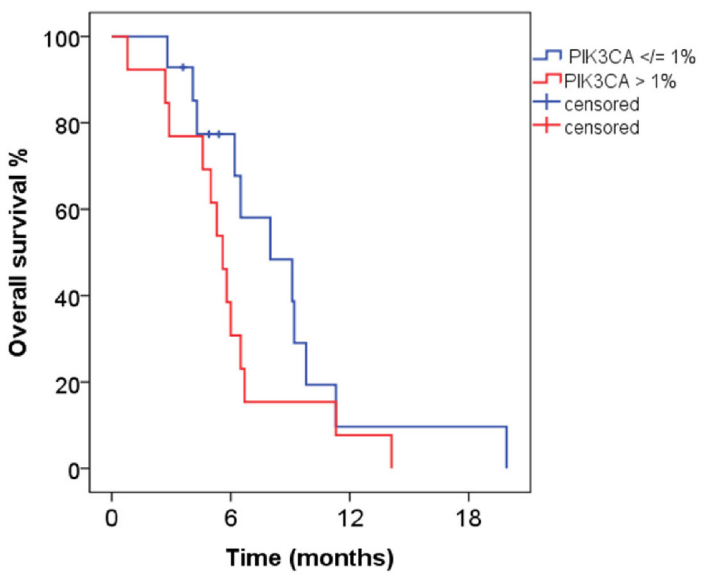

months, 95\% CI 4.5-10.1; $p=0.38$; Figure 1A). Of interest, 20 patients with $<=1 \%$ of $K R A S$-mutant cfDNA had a longer median survival compared to 41 patients with $>1 \%$ of KRAS-mutant cfDNA (7.3 months, $95 \%$ CI $5.3-9.3$ vs. 4.8 months, $95 \%$ CI $3.8-5.8 ; p=0.008$; Figure 1B). Finally, 14 patients with $<=1 \%$ of PIK3CAmutant cfDNA had a similar length of survival as did 13 patients with $>1 \%$ of PIK3CA-mutant cfDNA $(8.0$ months, $95 \%$ CI $4.0-12.0$ vs. 5.6 months, $95 \%$ CI $4.7-6.5$; $p=0.15$; Figure 1C). Survival analysis for patients with $E G F R$ mutations has not been performed due to the low number of patients in that group.

Next, we performed an analysis combining all tested mutations (BRAF, EGFR, KRAS, PIK3CA) in all of 105 patients with mutant cfDNA. When there was more than one mutation in the same patient, the mutation with the highest percentage of mutant DNA was used for analysis. Patients $(n=38)$ with $</=1 \%$ of mutant cfDNA had longer

B
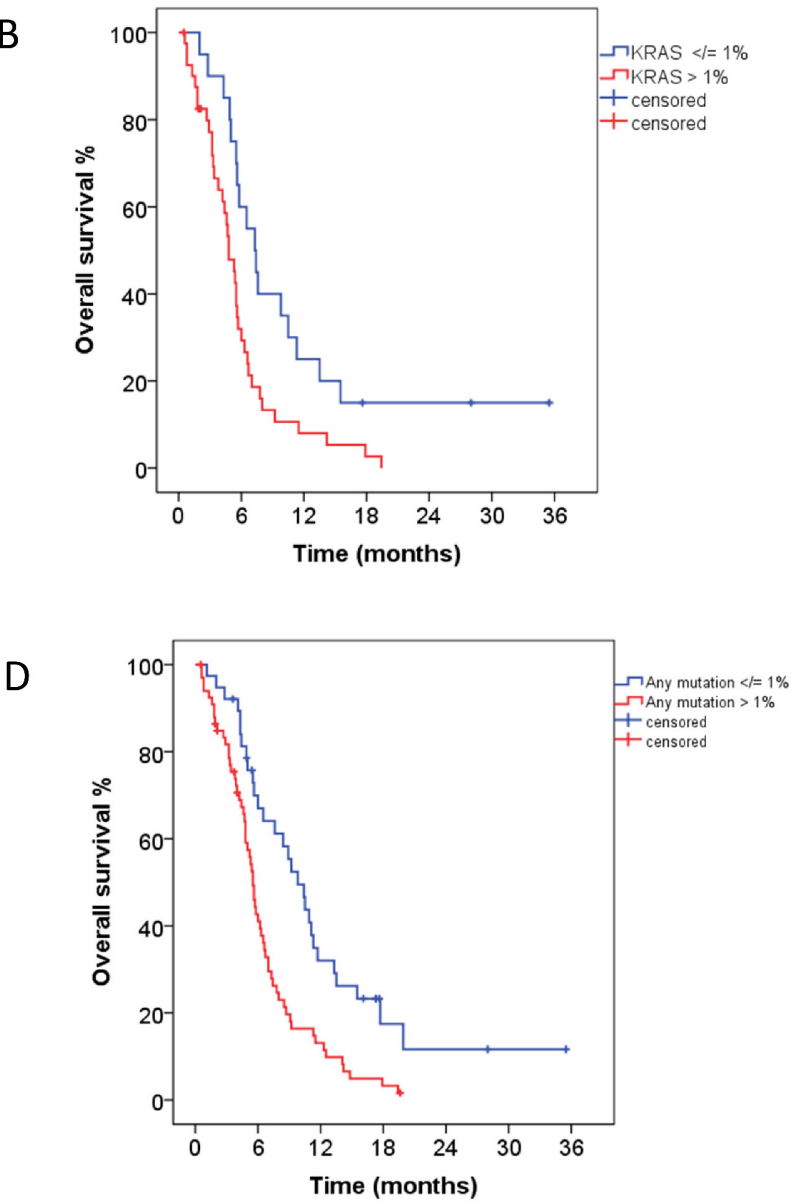

Figure 1: (A) In 33 patients with $B R A F$ mutations in cfDNA, 16 patients with $<=1 \%$ (blue) of $B R A F$ mutations had survival similar to 17 patients with $>1 \%$ (red) of $B R A F$ mutations (8.9 months, 95\% CI 7.3-10.5 vs. 7.3 months, 95\% CI 4.5-10.1; $p=0.38$ ). (B) In 61 patients with $K R A S$ mutations in cfDNA, 20 patients with $</=1 \%$ (blue) of $K R A S$ mutations had longer median survival compared to 41 patients with $>1 \%$ (red) of $K R A S$ mutations (7.3 months, $95 \%$ CI 5.3-9.3 vs. 4.8 months, 95\% CI 3.8-5.8; $p=0.008$ ). (C) In 27 patients with $P I K 3 C A$ mutations in cfDNA, 14 patients with $<=1 \%$ (blue) of $P I K 3 C A$ mutations had survival similar to 13 patients with $>1 \%$ (red) of PIK3CA mutations (8.0 months, 95\% CI 4.0-12.0 vs. 5.6 months, 95\% CI 4.7-6.5; $p=0.15$ ). (D) In 105 patients with $B R A F$, EGFR, KRAS, or PIK3CA mutations in cfDNA, 38 patients with $<=1 \%$ (blue) of mutant cfDNA had longer median survival compared to 67 patients with $>1 \%$ (red) of mutant cfDNA (9.8 months, 95\% CI 7.5-12.1 vs. 5.5 months, 95\% CI 5.0-6.0; $p=0.001)$. 
median survivals compared to 67 patients with $>1 \%$ of mutant cfDNA (9.8 months, 95\% CI $7.5-12.1$ vs. 5.5 months, 95\% CI 5.0-6.0; $p=0.001$; Figure 1D).

Finally, we analyzed the prognostic impact of cfDNA mutations on OS in multivariable analysis, which included the Royal Marsden Hospital prognostic score (RMH score) and the MD Anderson (MDACC) score. [23, 24] The RMH score is a prospectively validated tool used to predict OS in patients with advanced cancers referred for early-phase clinical trials. It is calculated on the basis of lactate dehydrogenase levels ( $>$ upper limit of normal vs. normal), albumin levels ( $<3.5 \mathrm{~g} / \mathrm{mL}$ vs. $3.5 \mathrm{~g} / \mathrm{mL}$ or higher $)$ and number of metastatic sites ( $>2$ sites vs. 2 sites or less) and scores of $0-1$ are associated with better survival than scores of 2-3. Similarly, the MDACC prognostic score included the factors listed above for the RMH score as well as ECOG performance status ( 0 vs. $>/=1)$ and type of tumor (gastrointestinal vs. other).

In 61 patients with KRAS mutations in cfDNA, 31 patients with $\mathrm{RMH}$ scores of $0-1$ had longer median

A

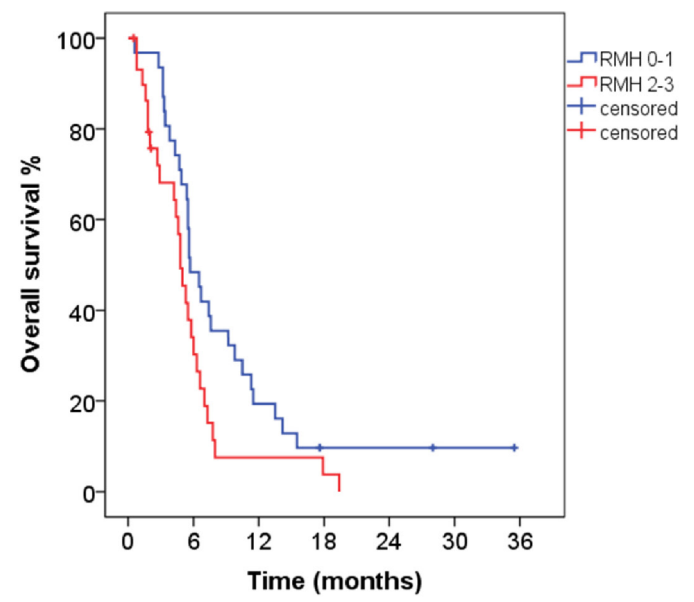

C

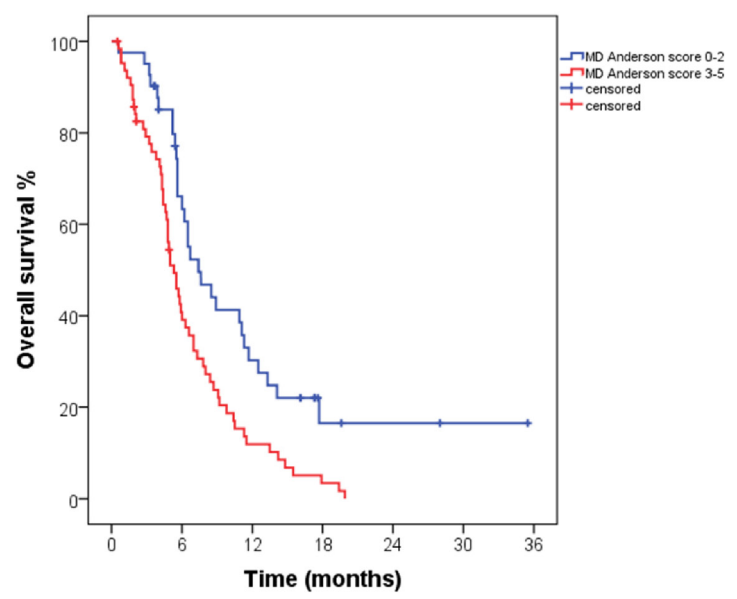

survivals than 30 patients with RMH scores of 2-3 (5.7 months, $95 \%$ CI $4.4-7.0$ vs. 4.8 months, 95\% CI 3.9-5.7; $p=$ 0.036 , Figure $2 \mathrm{~A})$. In multivariable analysis, which included $K R A S$ mutations in cfDNA $(</=1 \%$ vs. $>1 \%)$ and the $\mathrm{RMH}$ score ( $0-1$ vs. $2-3$ ), patients with KRAS mutations in $</=1 \%$ of cfDNA had a trend toward a longer survival compared to patients with KRAS mutations in $>1 \%$ of cfDNA (hazard ratio [HR] 0.53, 95\% CI 0.27-1.03, $p=0.06$ ).

In a combined analysis of 105 patients with cfDNA mutations, 57 patients with RMH scores of $0-1$ had longer median survivals than 48 patients with RMH scores of 2-3 (7.4 months, 95\% CI 4.9-9.9 vs. 5.3 months, 95\% CI $4.2-6.4 ; p=0.029$, Figure 2B). Similarly, 41 patients with MDACC scores of 0-2 had longer median survivals than 64 patients with MDACC scores of 3-5 (7.4 months, 95\% CI $4.5-10.3$ vs. 5.3 months, $95 \%$ CI $4.3-6.3 ; p=0.002$; Figure 2C). In multivariable analysis, which included mutant cfDNA $(</=1 \%$ vs. $>1 \%)$ and $\mathrm{RMH}$ score $(0-1$ vs. $2-3)$, patients with $</=1 \%$ of mutant cfDNA had a longer survival compared to patients with $>1 \%$ of mutant

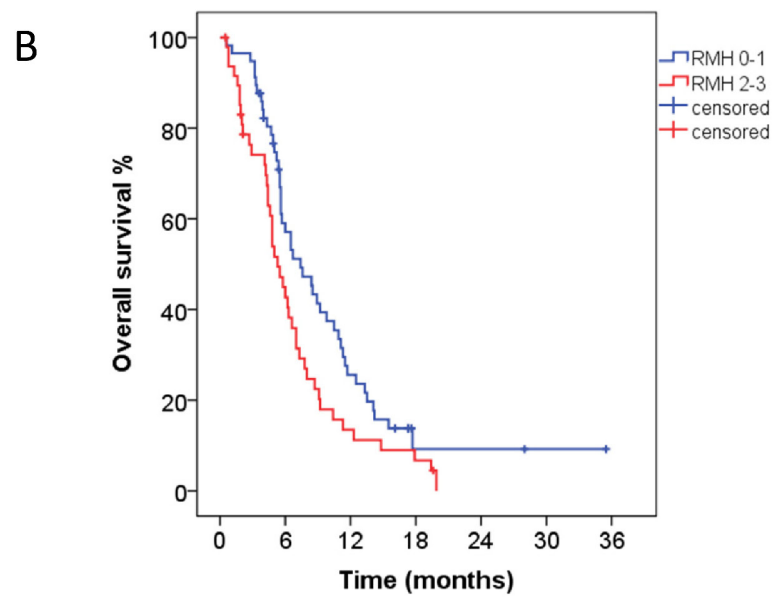

Figure 2: (A) In 61 patients with KRAS mutations in cfDNA, 31 patients with scores of $0-1$ had longer median survival than 30 patients with RMH scores of 2-3 (5.7 months, 95\% CI 4.4-7.0 vs. 4.8 months, 95\% CI 3.9-5.7; $p=0.036$ ). (B) In a combined analysis of 105 patients with any cfDNA mutation, 57 patients with RMH scores of $0-1$ had longer median survival than did 48 patients with RMH scores of 2-3 (7.4 months, 95\% CI 4.9-9.9 vs. 5.3 months, 95\% CI 4.2-6.4; $p=0.029)$. (C) In a combined analysis of 105 patients with any cfDNA mutation, 41 patients with MDACC scores of $0-2$ had longer median survival than 64 patients with MDACC scores of 3-5 (7.4 months, $95 \%$ CI $4.5-10.3$ vs. 5.3 months, $95 \%$ CI $4.3-6.3$; $p=0.002$ ). 
Table 4: Multivariable analysis of 105 patients with cfDNA mutations, which included mutant cfDNA $(</=1 \%$ vs. $>1 \%)$ and RMH score (0-1 vs. 2-3) or MDACC score (0-2 vs. 3-5)

\begin{tabular}{|c|c|c|c|c|}
\hline Outcome & Variable & Hazard ratio & 95\% Confidence interval & $P$ value \\
\hline \multirow{2}{*}{$\begin{array}{l}\text { Overall survival } \\
\text { (RMH model) }\end{array}$} & $\operatorname{cfDNA}(</=1 \%$ vs. $>1 \%)$ & 0.49 & $0.29-0.81$ & 0.005 \\
\hline & RMH score (0-1 vs. $2-3)$ & 0.87 & $0.55-1.39$ & 0.57 \\
\hline \multirow{2}{*}{$\begin{array}{l}\text { Overall survival } \\
\text { (MDACC model) }\end{array}$} & $\operatorname{cfDNA}(</=1 \%$ vs. $>1 \%)$ & 0.51 & $0.32-0.82$ & 0.005 \\
\hline & MDACC score (0-2 vs. $3-5)$ & 0.61 & $0.38-0.96$ & 0.033 \\
\hline
\end{tabular}

cfDNA (HR 0.49, 95\% CI 0.29-0.81, $p=0.005$ ). Similar results were obtained using the MDACC score (HR 0.51, $95 \%$ CI $0.32-0.82, p=0.005$, Table 4$)$.

\section{DISCUSSION}

In our study, we demonstrated that using the BEAMing technology, testing for 21 oncogenic mutations in $B R A F, E G F R, K R A S$ and PIK3CA genes in the plasma cfDNA of patients with advanced cancers referred for treatment with experimental targeted therapies, is feasible. In addition, testing of cfDNA demonstrated acceptable concordance (BRAF, 91\%; EGFR, 99\%; KRAS, 83\%; PIK3CA, 91\%) with standard of care mutation analysis of primary or metastatic tumor tissue obtained during clinical care. Higgins et al. [17] demonstrated a $100 \%$ concordance between using BEAMing to assess PIK3CA mutations in plasma cfDNA versus using BEAMing for PIK3CA mutations in the tumor tissue in a cohort of patients with advanced breast cancer when the plasma and tumor samples were obtained at the same time. However, the concordance decreased to $79 \%$ when tumor samples and plasma cfDNA were obtained at different time points. Board et al. [24] demonstrated a 95\% concordance between PIK3CA mutation status in plasma cfDNA and tumor tissue obtained at the same time by using an amplification refractory mutation system. Most recently, Thierry et al. [25] demonstrated a $96 \%$ concordance for combined $K R A S$ and $B R A F$ mutation testing using allelespecific quantitative PCR of plasma cfDNA compared to tissue (primary or metastatic) tested as standard of care. It is conceivable that mutation analysis results from cfDNA are highly concordant with mutation analysis results from tumor tissue if both materials are obtained concomitantly. However, the concordance rate can decrease, perhaps due to inherent heterogeneity, if both materials are obtained at different time points. This is not unexpected, since similar observations were made in a study in 33 matched primary and recurrent breast tumors, in which 97 of $112(86.6 \%)$ somatic mutations were concordant. [26] It is unclear, why our results demonstrated the lowest concordance for $K R A S$ compared to other genes ( $83 \%$ vs $91 \%-99 \%$ ) and whether this was related to underlying biology or technology (or both).
Detection of molecular aberrations in cfDNA can be also used to monitor response to therapy and emergence of secondary mutations associated with resistance to therapy, which can plausibly be used for therapeutic interventions. $[27,28]$ Because plasma cfDNA can originate from multiple tumor sites, arguably its molecular analysis may better reflect prevailing molecular aberrations than obtained from single-site biopsied tissue. [29, 30] In addition, unlike tissue biopsies, obtaining samples of cfDNA is a noninvasive approach, with less risk to patients at a lower cost. Diehl et al. [16], in a pilot study of 18 patients with metastatic colorectal cancer who were indicated as being candidates for surgical resection or radiofrequency ablation, showed that cfDNA from plasma samples can be isolated and oncogenic mutations $(A P C$, $K R A S, T P 53)$ can be detected in all tested patients using the BEAMing PCR-based technology. Further, analysis of a quantity of mutant copies more accurately predicted disease progression than standard evaluation of serum CEA levels. In a study of patients with metastatic breast cancer, $97 \%$ had genetic alterations in cfDNA and changes in cfDNA mutation levels correlated with changes in tumor burden to a greater degree than indicated by a CA 15-3 prognostic marker. Furthermore, two pilot studies in advanced colorectal cancer patients with wtKRAS demonstrated emerging $K R A S$ aberrations in cfDNA during treatment with an anti-EGFR therapy [31,32]. The first study published reported that $38 \%$ of patients treated with the anti-EGFR monoclonal antibody cetuximab, who were known to have wt KRAS on the basis of tumor tissue analysis, later developed KRAS mutations. These mutations were detectable in blood samples, usually between 5 and 6 months following treatment. [31] The second study, in patients who developed resistance to cetuximab or panitumumab, showed the emergence of $K R A S$ amplification in one sample and acquisition of secondary KRAS mutations in $60 \%$ of the cases. KRASmutant alleles were also detectable in the blood samples of cetuximab-treated patients as early as 10 months before disease progression appeared on restaging scans. [32] In our study we did not perform serial plasma collections at multiple time points; however, we noticed several interesting observations. For instance, we found in patients with NSCLC and EGFR mutations in the tumor tissue and prior therapy with EGFR inhibitors, secondary mutations 
(EGFR T790M and PIK3CA E545K) in plasma cfDNA or $K R A S$ or $B R A F$ mutations in the cfDNA of patients with colorectal cancer with wtKRAS in tumor tissue treated with EGFR antibodies, credibly explaining adaptive resistance to therapy.

Finally, it has been suggested that the presence and amount of mutant cfDNA can be associated with progression-free and OS. [16, 28, 33, 34] For instance, in a pivotal study, the absence of cfDNA in patients with colorectal cancer after surgical resection was associated with $100 \%$ recurrence-free survival. [16] Similarly, a higher amount of cfDNA and $K R A S$-mutant cfDNA found in patients with advanced colorectal cancer treated with irinotecan and cetuximab and in patients with advanced NSCLC treated with carboplatin and vinorelbine was associated with a shorter progression-free survival and OS. [33, 34] Finally, in a group of 206 patients with metastatic colorectal cancer, a higher concentration of cfDNA negatively correlated with OS. [28] In our study, a higher percentage of mutant cfDNA, irrespective of type of the mutation, was associated with a shorter OS (7.4 months vs. 5.3 months; $p=0.029$ ), which was confirmed in a multivariable analysis (HR $0.49,95 \% \mathrm{CI}$ $0.29-0.81, p=0.005)$. We made a similar observation in a separate analysis of patients with $K R A S$ mutations in cfDNA, which comprised the largest subgroup of our total patient population. Nevertheless, these results need to be interpreted cautiously and validated in future studies since they might have been influenced by tumor heterogeneity, the heterogeneity of our studied population and other factors.

In summary, we demonstrated that molecular analysis of cfDNA for selected oncogenic mutations in BRAF, EGFR, KRAS, and PIK3CA is feasible and demonstrates acceptable concordance with standard of care mutation testing of archival tumor tissue and that the amount of mutant cfDNA is an independent prognostic factor for survival. The possible impact of cfDNA mutations on survival must be interpreted with caution because of the retrospective nature of the study and the absence of a validation cohort. Furthermore, other factors such as tumor burden and proliferative activity were not assessed. Finally, even if a higher mutation burden predicts poor survival it remains unclear whether adding more effective therapies targeting underlying molecular aberrations and the tumor microenvironment might offset this effect. We also showed that mutations not originally found in the tumor tissue could be present at a low frequency in cfDNA, which can plausibly contribute to therapeutic resistance. In order to prove clinical utility, mutation analysis of cfDNA will need to be tested in prospective clinical trials, which will also include therapeutic intervention with respect to cfDNA mutation status. In addition, most of the sensitive technologies applicable for cfDNA testing, including BEAMing in our study, use PCR-based technologies, which limits simultaneous detection for multiple mutations. New technologies with high sensitivity and broad multiplex capability need to be developed to advance the results of analysis to the clinical arena.

\section{METHODS}

\section{Patients}

Starting in October 2010, patients with advanced cancers previously treated with standard therapies, who were previously tested for $B R A F$ and/or $E G F R$ and/or $K R A S$ and/or PIK3CA mutations in archival tumor tissue were enrolled in the study. Patients were required to be new referrals to the Department of Investigational Cancer Therapeutics as candidates for experimental therapies or potential patients had progressive disease if already treated with experimental therapies. The registration of patients in the database, tumor pathology assessment, and tumor mutation analysis were performed at MD Anderson. The study was conducted in accordance with MD Anderson Institutional Review Board guidelines.

\section{Tumor tissue analyses}

A total of 21 activating mutations in BRAF, EGFR, $K R A S$ and PIK3CA genes were investigated in archival tumor tissue obtained from routine clinical diagnostic and/or therapeutic procedures from primary or metastatic sites (Table 1). All histologies were centrally reviewed at MD Anderson. Mutation testing was performed in the CLIA-certified Molecular Diagnostic Laboratory within the Division of Pathology and Laboratory Medicine at MD Anderson. DNA was extracted from microdissected paraffin-embedded tumor sections and analyzed using a polymerase chain reaction-based DNA sequencing method for mutations outlined in Table 1 utilizing primers designed by the MD Anderson Molecular Diagnostic Laboratory. In January 2011, the assay was changed to mass spectrometric detection (Sequenom MassARRAY) and in March 2012, to next-generation sequencing (Ion Torrent, Life Technologies, Carlsbad, CA). The mutations identified during the initial screening were confirmed using a Sanger sequencing. The lower limit of detection is approximately $5-10 \%$ of the mutant allele frequency, which is influenced by clonal heterogeneity and the presence of normal tissue.

\section{Plasma cfDNA analyses}

Plasma samples used for cfDNA mutation analysis were obtained from whole blood collected in EDTA tubes, which was centrifuged and spun twice within 2 hours of collection. Isolation of cfDNA from plasma was carried out using the QIAamp DNA purification kit (Qiagen) and mutation analysis for a total of 21 mutations in BRAF, EGFR, KRAS, 
and PIK3CA (Table 1) with BEAMing assays were conducted on each sample by Inostics $\mathrm{GmbH}$ as previously published. [16-18] Briefly, individual DNA molecules were attached to magnetic beads in waterin-oil emulsions and then subjected to compartmentalized PCR amplification. The mutational status of DNA bound to beads was determined by hybridization to fluorescent allele-specific probes for mutant or wild-type (wt) of the gene of interest, respectively. Quantification of mutant DNA was performed using flow cytometry. Investigators performing mutation analysis of cfDNA with BEAMing were blinded to the results of mutation analysis of the archival tumor samples. The lower limit of detection is approximately $0.02 \%$ of mutant allele frequency.

\section{Statistical analysis}

Concordance between mutation analysis of archival tumor tissue and mutation analysis of cfDNA from plasma samples was calculated using a kappa coefficient. Concordance analyses were carried out using GraphPad Software (GraphPad Software, Inc.; La Jolla; CA). OS was defined as the time interval from the study entry to the date of death or the date of last follow up, whichever occurred first. OS was estimated using the method of Kaplan and Meier and compared among the subgroups of patients using a log-rank test. [19] Cox proportional hazards regression models were fit to assess the association between patient characteristics and OS. [20] All tests were two-sided, and $P$-values less than 0.05 were considered statistically significant. All statistical analyses were carried out using GraphPad Software (GraphPad Software, Inc.; La Jolla; CA) and SPSS 21 computer software (SPSS Chicago, IL).

\section{Explanation of authors contribution}

Study concept and design: FJ, PA, FD, RK.

Data collection and analysis: FJ, PA, VRH, GC.

Laboratory testing: PA, FD, RL.

Provision of patients and study materials: FJ, PA, AMT, SF, AN, GSF, DSH, JJW, SAP, RGZ, AYB, MJO, BKK, KBK, ESK, RL, FD, FMB, RK.

Manuscript writing and approval: FJ, PA, AMT, SF, AN, GSF, DSH, VRH, GC, JJW, SAP, RGZ, AYB, MJO, BKK, KBK, ESK, RL, FD, FMB, RK.

\section{ACKNOWLEDGMENTS}

We thank Ms. Joann Aaron for scientific review and editing of this article.

\section{CONFLICT OF INTEREST}

Filip Janku has research support from Novartis, Biocartis, Trovagene and Transgenomic. Philipp Angenendt and Frank Diehl are employees of Sysmex Inostics GmbH, Razelle Kurzrock has research support from EMD Serono. Funda Meric-Bernstam has research support from Novartis, Astra-Zeneca and has advisory/consulting roles with Novartis and Genentech.

\section{FINANCIAL SUPPORT}

Supported by Elsa U. Pardee Foundation, Sidney Kimmel Foundation for Cancer Research and the Sheikh Khalifa Al Nahyan Ben Zayed Institute for Personalized Cancer Therapy, NCATS grant UL1 TR000371 (Center for Clinical and Translational Sciences), and the MD Anderson Cancer Center Support grant (P30 CA016672).

\section{REFERENCES}

1. Druker BJ, Talpaz M, Resta DJ, Peng B, Buchdunger E, Ford JM, Lydon NB, Kantarjian H, Capdeville R, OhnoJones S, Sawyers CL. Efficacy and safety of a specific inhibitor of the BCR-ABL tyrosine kinase in chronic myeloid leukemia. N Engl J Med. 2001; 344:1031-1037.

2. Lynch TJ, Bell DW, Sordella R, Gurubhagavatula S, Okimoto RA, Brannigan BW, Harris PL, Haserlat SM, Supko JG, Haluska FG, Louis DN, Christiani DC, Settleman J, Haber DA. Activating mutations in the epidermal growth factor receptor underlying responsiveness of non-small-cell lung cancer to gefitinib. N Engl J Med. 2004; 350:2129-2139.

3. Kwak EL, Bang YJ, Camidge DR, Shaw AT, Solomon B, Maki RG, Ou SH, Dezube BJ, Janne PA, Costa DB, Varella-Garcia M, Kim WH, Lynch TJ, Fidias P, Stubbs H, Engelman JA, et al. Anaplastic lymphoma kinase inhibition in non-small-cell lung cancer. N Engl J Med. 2010; 363:1693-1703.

4. Falchook GS, Long GV, Kurzrock R, Kim KB, Arkenau TH, Brown MP, Hamid O, Infante JR, Millward M, Pavlick AC, O’Day SJ, Blackman SC, Curtis CM, Lebowitz P, Ma B, Ouellet D, et al. Dabrafenib in patients with melanoma, untreated brain metastases, and other solid tumours: a phase 1 dose-escalation trial. Lancet. 2012; 379:1893-1901.

5. Flaherty KT, Puzanov I, Kim KB, Ribas A, McArthur GA, Sosman JA, O'Dwyer PJ, Lee RJ, Grippo JF, Nolop K, Chapman PB. Inhibition of mutated, activated BRAF in metastatic melanoma. N Engl J Med. 2010; 363:809-819.

6. Tsimberidou AM, Iskander NG, Hong DS, Wheler JJ, Falchook GS, Fu S, Piha-Paul S, Naing A, Janku F, Luthra R, Ye Y, Wen S, Berry D, Kurzrock R. Personalized medicine in a phase I clinical trials program: the MD Anderson Cancer Center initiative. Clin Cancer Res. 2012; 18:6373-6383.

7. Demetri GD, von Mehren M, Blanke CD, Van den Abbeele AD, Eisenberg B, Roberts PJ, Heinrich MC, Tuveson DA, Singer S, Janicek M, Fletcher JA, Silverman SG, Silberman SL, Capdeville R, Kiese B, 
Peng B, et al. Efficacy and safety of imatinib mesylate in advanced gastrointestinal stromal tumors. N Engl J Med. 2002; 347:472-480.

8. Braiteh F, Kurzrock R. Uncommon tumors and exceptional therapies: paradox or paradigm? Mol Cancer Ther. 2007; 6:1175-1179.

9. Rosell R, Moran T, Queralt C, Porta R, Cardenal F, Camps C, Majem M, Lopez-Vivanco G, Isla D, Provencio M, Insa A, Massuti B, Gonzalez-Larriba JL, Paz-Ares L, Bover I, Garcia-Campelo R, et al. Screening for epidermal growth factor receptor mutations in lung cancer. N Engl J Med. 2009; 361:958-967.

10. Janku F, Tsimberidou AM, Garrido-Laguna I, Wang X, Luthra R, Hong DS, Naing A, Falchook GS, Moroney JW, Piha-Paul SA, Wheler JJ, Moulder SL, Fu S, Kurzrock R. PIK3CA mutations in patients with advanced cancers treated with PI3K/AKT/mTOR axis inhibitors. Molecular cancer therapeutics. 2011; 10:558-565.

11. Dupont Jensen J, Laenkholm AV, Knoop A, Ewertz M, Bandaru R, Liu W, Hackl W, Barrett JC, Gardner H. PIK3CA mutations may be discordant between primary and corresponding metastatic disease in breast cancer. Clin Cancer Res. 2011; 17:667-677.

12. Gonzalez-Angulo AM, Ferrer-Lozano J, Stemke-Hale K, Sahin A, Liu S, Barrera JA, Burgues O, Lluch AM, Chen H, Hortobagyi GN, Mills GB, Meric-Bernstam F. PI3K pathway mutations and PTEN levels in primary and metastatic breast cancer. Mol Cancer Ther. 2011; 10:1093-1101.

13. Sequist LV, Waltman BA, Dias-Santagata D, Digumarthy S, Turke AB, Fidias $\mathrm{P}$, Bergethon $\mathrm{K}$, Shaw AT, Gettinger S, Cosper AK, Akhavanfard S, Heist RS, Temel J, Christensen JG, Wain JC, Lynch TJ, et al. Genotypic and Histological Evolution of Lung Cancers Acquiring Resistance to EGFR Inhibitors. Sci Transl Med. 2011; 3:75ra26.

14. Turner NC, Reis-Filho JS. Genetic heterogeneity and cancer drug resistance. Lancet Oncol. 2012; 13:e178-185.

15. Gerlinger M, Rowan AJ, Horswell S, Larkin J, Endesfelder D, Gronroos E, Martinez P, Matthews N, Stewart A, Tarpey P, Varela I, Phillimore B, Begum S, McDonald NQ, Butler A, Jones D, et al. Intratumor heterogeneity and branched evolution revealed by multiregion sequencing. N Engl J Med. 2012; 366:883-892.

16. Diehl F, Schmidt K, Choti MA, Romans K, Goodman S, Li M, Thornton K, Agrawal N, Sokoll L, Szabo SA, Kinzler KW, Vogelstein B, Diaz LA Jr. Circulating mutant DNA to assess tumor dynamics. Nat Med. 2008; 14:985-990.

17. Higgins MJ, Jelovac D, Barnathan E, Blair B, Slater S, Powers P, Zorzi J, Jeter SC, Oliver GR, Fetting J, Emens L, Riley C, Stearns V, Diehl F, Angenendt P, Huang P, et al. Detection of tumor PIK3CA status in metastatic breast cancer using peripheral blood. Clinical cancer research: an official journal of the American Association for Cancer Research. 2012; 18:3462-3469.
18. Diehl F, Li M, He Y, Kinzler KW, Vogelstein B, Dressman D. BEAMing: single-molecule PCR on microparticles in water-in-oil emulsions. Nat Methods. 2006; 3:551-559.

19. Kaplan EM. Nonparametric estimator from incomplete observations. J American Statistical Association. 1958; 53:457-481.

20. Cox D. Regression models and life tables (with discussion). J R Statistical Soc, B. 1972; 34:187-220.

21. Janku F, Hong DS, Fu S, Piha-Paul SA, Naing A, Falchook GS, Tsimberidou AM, Stepanek VM, Moulder SL, Lee JJ, Luthra R, Zinner RG, Broaddus RR, Wheler JJ, Kurzrock R. Assessing PIK3CA and PTEN in Early-Phase Trials with PI3K/AKT/ mTOR Inhibitors. Cell Rep. 2014; 6:377-387.

22. Janku F, Wheler JJ, Naing A, Falchook GS, Hong DS, Stepanek VM, Fu S, Piha-Paul SA, Lee JJ, Luthra R, Tsimberidou AM, Kurzrock R. PIK3CA mutation H1047R is associated with response to $\mathrm{PI} 3 \mathrm{~K} / \mathrm{AKT} / \mathrm{mTOR}$ signaling pathway inhibitors in early-phase clinical trials. Cancer Res. 2013; 73:276-284.

23. Arkenau HT, Barriuso J, Olmos D, Ang JE, de Bono J, Judson I, Kaye S. Prospective validation of a prognostic score to improve patient selection for oncology phase I trials. J Clin Oncol. 2009; 27:2692-2696.

24. Board RE, Wardley AM, Dixon JM, Armstrong AC, Howell S, Renshaw L, Donald E, Greystoke A, Ranson M, Hughes A, Dive C. Detection of PIK3CA mutations in circulating free DNA in patients with breast cancer. Breast Cancer Res Treat. 2010; 120:461-467.

25. Thierry AR, Mouliere F, El Messaoudi S, Mollevi C, LopezCrapez E, Rolet F, Gillet B, Gongora C, Dechelotte P, Robert B, Del Rio M, Lamy PJ, Bibeau F, Nouaille M, Loriot V, Jarrousse AS, et al. Clinical validation of the detection of KRAS and BRAF mutations from circulating tumor DNA. Nat Med. 2014; 20:430-435.

26. Meric-Bernstam F, Frampton GM, Ferrer-Lozano J, Yelensky R, Perez-Fidalgo JA, Wang Y, Palmer GA, Ross JS, Miller VA, Su X, Eroles P, Barrera JA, Burgues O, Lluch AM, Zheng X, Sahin A, et al. Concordance of Genomic Alterations between Primary and Recurrent Breast Cancer. Mol Cancer Ther. 2014.

27. Misale S, Arena S, Lamba S, Siravegna G, Lallo A, Hobor S, Russo M, Buscarino M, Lazzari L, SartoreBianchi A, Bencardino K, Amatu A, Lauricella C, Valtorta E, Siena S, Di Nicolantonio F, et al. Blockade of EGFR and MEK intercepts heterogeneous mechanisms of acquired resistance to anti-EGFR therapies in colorectal cancer. Sci Transl Med. 2014; 6:224ra226.

28. Bettegowda C, Sausen M, Leary RJ, Kinde I, Wang Y, Agrawal N, Bartlett BR, Wang H, Luber B, Alani RM, Antonarakis ES, Azad NS, Bardelli A, Brem H, Cameron JL, Lee CC, et al. Detection of circulating tumor DNA in early- and late-stage human malignancies. Sci Transl Med. 2014; 6:224ra224.

29. Forshew T, Murtaza M, Parkinson C, Gale D, Tsui DW, Kaper F, Dawson SJ, Piskorz AM, Jimenez-Linan M, 
Bentley D, Hadfield J, May AP, Caldas C, Brenton JD, Rosenfeld N. Noninvasive identification and monitoring of cancer mutations by targeted deep sequencing of plasma DNA. Science translational medicine. 2012; 4(136):136ra168.

30. Murtaza M, Dawson SJ, Tsui DW, Gale D, Forshew T, Piskorz AM, Parkinson C, Chin SF, Kingsbury Z, Wong AS, Marass F, Humphray S, Hadfield J, Bentley D, Chin TM, Brenton JD, et al. Non-invasive analysis of acquired resistance to cancer therapy by sequencing of plasma DNA. Nature. 2013; 497:108-112.

31. Diaz LA Jr., Williams RT, Wu J, Kinde I, Hecht JR, Berlin J, Allen B, Bozic I, Reiter JG, Nowak MA, Kinzler KW, Oliner KS, Vogelstein B. The molecular evolution of acquired resistance to targeted EGFR blockade in colorectal cancers. Nature. 2012; 486:537-540.
32. Misale S, Yaeger R, Hobor S, Scala E, Janakiraman M, Liska D, Valtorta E, Schiavo R, Buscarino M, Siravegna G, Bencardino K, Cercek A, Chen CT, Veronese S, Zanon C, Sartore-Bianchi A, et al. Emergence of KRAS mutations and acquired resistance to anti-EGFR therapy in colorectal cancer. Nature. 2012; 486:532-536.

33. Spindler KL, Pallisgaard N, Vogelius I, Jakobsen A. Quantitative cell-free DNA, KRAS, and BRAF mutations in plasma from patients with metastatic colorectal cancer during treatment with cetuximab and irinotecan. Clin Cancer Res. 2012; 18:1177-1185.

34. Nygaard AD, Garm Spindler KL, Pallisgaard N, Andersen RF, Jakobsen A. The prognostic value of KRAS mutated plasma DNA in advanced non-small cell lung cancer. Lung Cancer. 2013; 79:312-317. 